\title{
Percepción de pacientes frente a la atención odontológica ofrecida por estudiantes en una universidad pública colombiana
}

\section{Patients' perceptions of dental care provided by students at a Colombian public university}

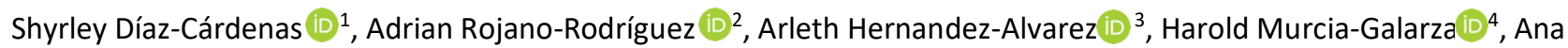 \\ Ardila-Torres (1D $)^{5}$
}

1. Universidad de Cartagena. Cartagena de Indias, Colombia. Correo: sdiazc@unicartagena.edu.co - https://orcid.org/0000-0003-1967-8981

2. Universidad de Cartagena. Cartagena de Indias, Colombia. Correo: arojanor@unicartagena.edu.co - https://orcid.org/0000-0001-6465-7886

3. Universidad de Cartagena. Cartagena de Indias, Colombia. Correo: ahernandeza1@unicartagena.edu.co - https://orcid.org/0000-0002-6920-2386

4. Universidad de Cartagena. Cartagena de Indias, Colombia. Correo: murcia_galarz@hotmail.com - https://orcid.org/0000-0003-4292-4984

5. Universidad de Cartagena. Cartagena de Indias, Colombia. Correo: ardila_2708@hotmail.com - https://orcid.org/0000-0002-6734-2211

Tipología: Artículo de investigación científica y tecnológica

Para citar este artículo: Díaz-Cárdenas S, Rojano-Rodríguez A, Hernández-Álvarez A, Murcia-Galarza H, Ardila-Torres A. Percepción de los pacientes frente a la atención odontológica ofrecida por estudiantes en una universidad pública colombiana. Duazary. 2021 abril; 18(2): 163-174. Doi: https://doi.org/10.21676/2389783X.4073

Recibido en mayo 18 de 2020

Aceptado en febrero 24 de 2021

Publicado en línea en mayo 25 de 2021

\section{RESUMEN}

Palabras

clave: percepción; pacientes; atención odontológica; estudiantes de odontología.
El objetivo de la investigación fue conocer la percepción de pacientes de la atención odontológica ofrecida por estudiantes. Se realizó un estudio descriptivo longitudinal en 123 binomios pacienteestudiante en una escuela de odontología de una universidad pública colombiana, a los cuales se les aplicó cuestionario para indagar variables sociodemográficas, la atención odontológica y factores relacionados con la percepción del paciente - presentación personal del estudiante, interacción del estudiante-paciente y transmisión de conocimientos del estudiante-paciente- durante dos momentos de la consulta: al inicio (T0) y a los dos meses (T1). Se hallaron diferencias entre T0 y T1, con aumento en las frecuencias relacionadas con la atención odontológica, y el factor interacción estudiante-paciente fue el más influyente. Al transcurrir el semestre y según el sexo del estudiante, la percepción de la atención odontológica ofrecida se comporta mejor en T1. Se concluye que la percepción de los pacientes frente a la atención odontológica ofrecida por estudiantes se encuentra influenciada por factores propios de estos como el sexo al que pertenece y el semestre, reflejándose directamente en la interacción estudiante-paciente, que conlleva a satisfacción de la atención recibida y éxitos en los tratamientos odontológicos realizados.

\section{ABSTRACT}

Keywords:

Perception; Patients; Dental care; Students, Dental.
The objective of the study was to know the perception of patients of the dental care offered by students. A longitudinal descriptive study was carried out in 123 patient-student binomials in a dental school of a Colombian public university, to which a questionnaire was applied to investigate sociodemographic variables, dental care and factors related to the patient's perception: Personal presentation of the student, student-patient interaction and student-patient knowledge transmission during two moments of the attention: at the beginning (TO) and after two months (T1). Differences were found between T0 and T1 with an increase between the frequencies related to dental care; the student-patient interaction factor was the most influential. As the semester elapses and according to the student's sex, the perception behaves better in $\mathrm{T} 1$ of the dental care offered. It is concluded that the perception of patients 
Percepción de pacientes frente a la atención odontológica ofrecida por estudiantes en una universidad pública colombiana

regarding the dental care offered by students is influenced by student's own factors such as sex and semester, directly reflecting on the student-patient interaction that leads to satisfaction of the care received and successes in dental treatments performed.

\section{INTRODUCCIÓN}

La atención recibida y ofertada en los servicios de la salud se encuentra directamente relacionada con la percepción de cada paciente, la cual varía a causa de diversos factores que pueden ser propios de cada individuo (internos) como relacionados con quienes brindan el servicio. En este segundo caso se encuentran factores tales como la cordialidad y la simpatía de todo el personal de salud que proporciona la atención, la facilidad para acudir donde el prestador, características de las instalaciones (recepción, sala de espera, baños), duración y simplicidad para acceder a una cita, los resultados del tratamiento recibido y el manejo de complicaciones durante el tratamiento, los cuales van a influir y determinar la calidad de la atención recibida por parte del usuario ${ }^{1-3}$.

En este sentido adquiere importancia la percepción que presentan los pacientes que acuden a diferentes programas de salud de universidades como las facultades de Odontología, en especial porque el servicio es ofertado por personas en formación, lo que representa una mayor responsabilidad y, por ende, un mayor reto debido a que su condición de estudiante podría llevar a un paciente a suponer que no cuenta con una suficiente experticia en la atención odontológica. En todo caso, se espera que los conocimientos y la capacitación que el estudiante ha adquirido a lo largo de su carrera, de manera conjunta con la práctica diaria, se reflejen a la hora de llevar a cabo sus prácticas y en la atención en sus diferentes ámbitos ${ }^{4}$. De tal manera, el conocimiento, la práctica y la ética profesional son componentes esenciales e indispensables de todos los profesionales de la salud o estudiantes en prácticas cuando brindan y ofertan alternativas de tratamiento a usuarios que acuden, en calidad de pacientes, a clínicas de las facultades de Odontología de una institución de educación superior $^{5,6}$.

Estar al tanto de la percepción que presenta el paciente en cualquier momento del desarrollo de la atención brindada le permite al prestador conocer, entender e identificar las necesidades de los pacientes y mejorar como institución, buscando siempre brindar una atención de calidad con la cual el usuario sienta satisfacción ${ }^{7}$. Además, dicha información puede servir como guía determinante para mejorar factores que generen un ambiente confiable a los pacientes que acuden a la facultad $y$, de algún modo, aportarle datos a la universidad para fortalecer la capacidad instalada en el mejoramiento continuo en el área de odontología, manteniendo así la simbiosis entre estudiante de odontología, paciente y Facultad de Odontología, que a futuro y a lo sumo se podrá ver reflejada en la calidad del futuro profesional.

No obstante, en su gran mayoría, las investigaciones realizadas en la materia muestran la percepción de pacientes sobre la atención recibida por parte de profesionales ya egresados del campo de la odontología en instituciones prestadoras de servicios de salud públicas y privadas. Por otra parte, son muy pocos los estudios en donde se reporta esta percepción de los pacientes cuando han sido atendidos por estudiantes de odontología en su práctica académica-clínica ${ }^{8,9}$. Por lo tanto, el objetivo del trabajo discutido en este artículo consistió en identificar la percepción de los pacientes frente a la atención odontológica ofrecida por estudiantes en una universidad pública colombiana.

\section{MATERIALES Y MÉTODOS}

\section{Tipo de investigación}

Estudio de tipo descriptivo longitudinal.

\section{Participantes}

La población universo corresponde a estudiantes de odontología de la ciudad de Cartagena pertenecientes a una universidad pública. De aquí se tomaron aquellos que se encontraban en áreas clínicas, de forma que, en definitiva, participaron 
123 binomios estudiante-paciente que acudieron a la Clínica Integral del Adulto. Los estudiantes se encontraban cursando octavo, noveno o décimo semestre del primer periodo académico 2017.

Como criterio de inclusión se consideró que los participantes aceptaran de manera libre y voluntaria formar parte del estudio. Se excluyeron pacientes con problemas cognitivos, discapacidad motora o enfermedades crónicas avanzadas que impidieran el estudio. Para el cálculo de la muestra se asumió una prevalencia del evento esperado (satisfacción del usuario) del $90 \%$ según lo recomendado para realizar este tipo de estudios y un error estándar del $5 \%$. El tipo de muestreo utilizado fue aleatorio simple.

\section{Instrumentos}

Se diseñó y aplicó un instrumento tipo cuestionario autodiligenciable, el cual fue revisado previamente en una prueba piloto realizada en pacientes que presentaban características similares a la población de estudio en otra clínica odontológica universitaria ${ }^{5}$. El cuestionario se encuentra dividido en dos partes: el primer segmento identifica las variables sociodemográficas del estudiante como edad (adulto joven [20-44 años], maduro [45-59 años]), sexo (masculino, femenino), estado civil (soltero, casado), ingresos socioeconómicos (bajo, medio, alto) y semestre cursado (octavo, noveno, décimo). La segunda parte, diligenciada por el paciente, permite identificar las variables sociodemográficas de este como edad (adulto joven [20-44 años], maduro [45-59 años] o mayor [ $\geq 60$ años]), sexo (masculino, femenino), estado civil (soltero, casado), religión (católico, cristiano, evangélico, testigo de jehová), escolaridad (bajo, medio, alto), ingresos socioeconómicos (bajo, medio, alto) y preguntas relacionadas con la atención general del servicio odontológico. A su vez, se incluyeron los factores que pueden hacer variar la percepción del paciente sobre la atención odontológica ofrecida por el estudiante, tales como: 1) presentación personal del estudiante, 2) interacción del estudiante con el paciente, y 3) transmisión de conocimientos del estudiante al paciente. Estos factores se clasificaron de esta forma luego de la revisión bibliográfica exhaustiva con respecto a la temática estudiada ${ }^{3,10}$.
Las preguntas fueron dicotómicas y politómicas, y aquellas relacionadas con la percepción fueron evaluadas según escala tipo Likert con opciones de "siempre", "casi siempre", "algunas veces", "casi nunca" y "nunca", con un valor de cinco a cero respectivamente. Se aplicó la prueba alfa de Cronbach para obtener la consistencia interna del instrumento y se obtuvo como resultado 0,82 .

\section{Procedimiento}

En el segundo semestre del año 2017 se aplicó el cuestionario. Para ello se emplearon dos tiempos diferentes en el desarrollo de la institución: a los dos meses de inicio de la Clínica del Adulto Integral (tiempo cero, T0) y a los dos meses de la primera recolección (tiempo uno, T1). La encuesta fue aplicada por personas ajenas al estudio, pero previamente calibradas y entrenadas para ello.

En primer lugar, se le entregaba el instrumento al estudiante para que diligenciara lo relacionado a las variables sociodemográficas, y posteriormente este indicaba quién era su paciente. Este último se ubicaba entonces en la sala de espera para diligenciar lo que le correspondía, evitando así que se sintiera intimidado o presionado ante la presencia del estudiante de odontología que lo atendía (TO). Luego se repitió este proceso solo con el paciente, dos meses después de la primera encuesta (T1).

\section{Análisis estadístico}

La base de datos se construyó en Microsoft Excel $2007^{\circledR}$, y el análisis de los datos se llevó a cabo en el programa estadístico Stata $X^{\circledR}$, licencia 1990540768. Se estimaron frecuencias y proporciones de variables sociodemográficas y de variables relacionadas con la atención del servicio odontológico, y se compararon entre el T0 y el T1. Asimismo, se hicieron sumatorias de respuestas para cada una de las preguntas relacionadas con la percepción del paciente sobre la atención odontológica ofrecida por el estudiante, y luego se estableció la relación entre dicha percepción y las variables de estudio a través de la prueba $T$ de Student para determinar su significancia. 


\section{Declaración sobre aspectos éticos}

Todos los participantes fueron debidamente informados y firmaron consentimiento para participar en el estudio. Esta investigación adaptó las normas establecidas en la Declaración de Helsinki de 1975 y en la Resolución 8430 de 1993 del Ministerio de Protección Social de Colombia. Se considera un estudio sin riesgo debido a que no se realiza ninguna intervención o modificación intencionada de las variables biológicas, fisiológicas, psicológicas o sociales de los individuos que participan en el estudio. La información recolectada es de carácter confidencial, por lo que no se dará a conocer la identidad de los participantes, respetando así su dignidad, la protección de sus derechos y su bienestar. Además, se contó con el aval del Departamento de Investigaciones de la Facultad de Odontología donde se realizó el estudio

\section{RESULTADOS}

Con relación a características sociodemográficas de los pacientes, se puede destacar que las instalaciones de las clínicas de la Facultad de Odontología son visitadas con mayor frecuencia por usuarios pertenecientes al ciclo vital de adulto joven $(35,7 \%)$, del sexo femenino $(59,4 \%)$, de estrato socioeconómico medio $(65,8 \%)$ y con un nivel de escolaridad medio (25,2\%). En cuanto a los estudiantes, se observa que la mayoría se encuentran en el ciclo vital de adulto joven (87,9\%), más de la mitad corresponden al sexo femenino $(68,6 \%)$, la mayor cantidad pertenece a noveno semestre $(36,1 \%)$, es decir, semestre clínico, y se encuentran en estrato socioeconómico medio $(56,6 \%)$ (Tabla 1$)$.

En cuanto a la atención general del servicio odontológico recibido en la facultad, se hallaron diferencias entre las respuestas al inicio (TO) y al final del semestre (T1), con aumento entre las frecuencias. Lo mismo sucedió al indagar por los tratamientos odontológicos y por la relación paciente-estudiante (Tabla 2).

Dentro de las variables relacionadas con la percepción del paciente sobre la atención odontológica ofrecida por el estudiante, específicamente en lo que corresponde a la presentación personal de este, el uso de las barreras de bioseguridad a nivel de la unidad odontológica fue la que mejores valores presentó $(94,1 \%)$ (Tabla $3)$. Respecto a la interacción del estudiante con el paciente, las variables que mejor se comportan corresponden al trato amigable, gentil y respetuoso durante la atención (96,5\%), y la satisfacción que presenta el paciente por la atención recibida hasta la fecha de realización de la encuesta $(96,8 \%$ ) (tabla $3)$. En cuanto a los factores relacionados con la transmisión de conocimientos, los pacientes concluyen que el estudiante mostró seguridad durante el procedimiento que le estuvo realizando mientras estaba solo sin el docente $(90,6 \%)$, y además se aprecia que el estudiante explica al paciente correctamente el procedimiento que realizará, convirtiéndose en la variable con mejores valores dentro de este factor $(92,4 \%)$ (Tabla 3$)$.

Al comparar la percepción de la atención recibida en T0 y T1 se observa una mejoría en los resultados de los pacientes tratados en el servicio odontológico de la universidad hasta llegar a excelente durante el transcurso académico del semestre (Figura 1).

Al comparar y apreciar con respecto al sexo, se evidenció una mejor percepción inicial (TO) en pacientes de sexo femenino (Figura $2 \mathrm{~A}$ ), la cual se mantuvo en el tiempo uno (T1) (Figura 2B).

Por otro lado, se evidencia que los pacientes consideran que reciben mejor atención cuando son tratados por estudiantes de décimo semestre en TO (Figura 3A). Esta percepción se mantiene en T1 (Figura 3B). 
Tabla 1. Distribución de las variables sociodemográficas.

\begin{tabular}{|l|c|c|}
\hline \multicolumn{1}{|c|}{ Variables sociodemográficas } & $\begin{array}{c}\text { Pacientes } \\
\text { Frecuencia (\%) }\end{array}$ & $\begin{array}{c}\text { Estudiantes } \\
\text { Frecuencia (\%) }\end{array}$ \\
\hline Edad & & $73(87,9)$ \\
\hline Adulto joven (20-44 años) & $44(35,7)$ & $10(12,1)$ \\
\hline Adulto maduro (45-59 años) & $37(30)$ & - \\
\hline Adulto mayor ( $\geq 60$ años) & $42(34,3)$ & $26(31,3)$ \\
\hline Sexo & & $57(68,6)$ \\
\hline Masculino & $50(40,7)$ & $24(28,9)$ \\
\hline Femenino & $73(59,4)$ & $47(56,6)$ \\
\hline Estrato socioeconómico & & $12(14,4)$ \\
\hline Bajo & $33(26,8)$ & \\
\hline Medio & $81(65,8)$ & - \\
\hline Alto & $9(7,3)$ & \\
\hline Nivel de escolaridad & & \\
\hline Bajo & $80(65,1)$ & - \\
\hline Medio & $31(25,2)$ & \\
\hline Alto & $10(8,1)$ & \\
\hline Semestre & & $27(32,5)$ \\
\hline Octavo & - & $30(36,1)$ \\
\hline Noveno & - & $26(31,3)$ \\
\hline Décimo & - & \\
\hline
\end{tabular}

Tabla 2. Distribución de los resultados con respecto a las preguntas relacionadas con la atención general del servicio odontológico.

\begin{tabular}{|c|c|c|c|c|c|c|c|c|}
\hline $\begin{array}{l}\text { Variables relacionadas con el } \\
\text { servicio odontológico }\end{array}$ & & $\begin{array}{r}\mathrm{T} \\
\text { Frecuen }\end{array}$ & (\%) & & & $\begin{array}{r}\text { T1 } \\
\text { Frecuenc }\end{array}$ & a (\%) & \\
\hline Puntuaciones & Excelente & Bueno & Regular & Malo & Excelente & Bueno & Regular & Malo \\
\hline $\begin{array}{l}\text { ¿Cómo calificaría la atención } \\
\text { que usted recibe en la } \\
\text { Facultad de Odontología? }\end{array}$ & $46(37,40)$ & $\begin{array}{c}44 \\
(35,77)\end{array}$ & $\begin{array}{c}33 \\
(26,83)\end{array}$ & 0 & $\begin{array}{c}80 \\
(65,04)\end{array}$ & $\begin{array}{c}42 \\
(34,15)\end{array}$ & $1(0,81)$ & 0 \\
\hline $\begin{array}{l}\text { ¿Le han comentado cuáles son } \\
\text { los tratamientos en la } \\
\text { Facultad de Odontología? }\end{array}$ & $45(36,59)$ & $\begin{array}{c}41 \\
(33,33)\end{array}$ & $\begin{array}{c}37 \\
(30,08)\end{array}$ & 0 & $\begin{array}{c}76 \\
(61,79)\end{array}$ & $\begin{array}{c}46 \\
(37,40)\end{array}$ & $1(0,81)$ & 0 \\
\hline $\begin{array}{l}\text { ¿Cómo calificaría la relación } \\
\text { entre el paciente y el } \\
\text { estudiante de manera } \\
\text { general? }\end{array}$ & $66(53,66)$ & $\begin{array}{c}41 \\
(33,33)\end{array}$ & $\begin{array}{c}16 \\
(13,01)\end{array}$ & 0 & $\begin{array}{c}84 \\
(68,29)\end{array}$ & $\begin{array}{c}37 \\
(30,08)\end{array}$ & $2(1,63)$ & 0 \\
\hline
\end{tabular}


Percepción de pacientes frente a la atención odontológica ofrecida por estudiantes en una universidad pública colombiana

Tabla 3. Comparación de sumatoria de respuestas frente a cada uno de los factores relacionados con la percepción del paciente sobre a la atención odontológica ofrecida por el estudiante.

\begin{tabular}{|c|c|c|}
\hline Factores relacionados con la presentación personal del estudiante & $\begin{array}{c}\text { T0 } \\
\text { Frecuencia } \\
\text { (\%) }\end{array}$ & $\begin{array}{c}\mathrm{T} 1 \\
\text { Frecuencia (\%) }\end{array}$ \\
\hline $\begin{array}{l}\text { ¿Usted posee una buena percepción en relación con la atención brindada por } \\
\text { el estudiante? }\end{array}$ & $485(75,5)$ & $571(92,8)$ \\
\hline $\begin{array}{l}\text { ¿Tiene en cuenta la presentación personal del estudiante al ser atendido por } \\
\text { este? }\end{array}$ & $504(82,6)$ & $560(91,0)$ \\
\hline $\begin{array}{l}\text { ¿Usted posee una buena percepción en relación con la atención brindada por } \\
\text { el profesor? }\end{array}$ & $473(75,9)$ & $563(92,2)$ \\
\hline ¿Usa el estudiante de odontología la protección de bioseguridad al atenderlo? & $548(89,8)$ & $541(94,1)$ \\
\hline ¿El estudiante mantiene en orden el instrumental y el cubículo donde trabaja? & $475(77,8)$ & $566(92,7)$ \\
\hline $\begin{array}{l}\text { ¿Los instrumentos y los equipos que son utilizados en su atención son de } \\
\text { buena calidad? }\end{array}$ & $527(86,3)$ & $560(91,8)$ \\
\hline $\begin{array}{l}\text { ¿El estudiante se encuentra bien afeitado o, si es mujer, se encuentra bien } \\
\text { arreglada? }\end{array}$ & $545(89,3)$ & $564(92,4)$ \\
\hline $\begin{array}{l}\text { ¿Notó usted que el estudiante prestó instrumental a algún compañero durante } \\
\text { la consulta? }\end{array}$ & $192(31,4)$ & $126(20,6)$ \\
\hline $\begin{array}{l}\text { ¿El estudiante contestó el celular o realizó otra actividad diferente a atenderlo } \\
\text { con los guantes puestos? }\end{array}$ & $152(24,9)$ & $125(20,4)$ \\
\hline Factores relacionados con la interacción paciente-estudiante & $\begin{array}{c}\text { T0 } \\
\text { Frecuencia } \\
\text { (\%) }\end{array}$ & $\begin{array}{c}\text { T1 } \\
\text { Frecuencia (\%) }\end{array}$ \\
\hline $\begin{array}{l}\text { ¿El estudiante de odontología se comportó de manera amigable, gentil y } \\
\text { respetuosa al atenderlo? }\end{array}$ & $543(89,0)$ & $589(96,5)$ \\
\hline $\begin{array}{l}\text { ¿Se encuentra satisfecho a la fecha con la atención recibida en la Facultad de } \\
\text { Odontología? }\end{array}$ & $569(93,2)$ & $591(96,8)$ \\
\hline $\begin{array}{l}\text { ¿Considera adecuada la privacidad manejada por el estudiante que lo atendió } \\
\text { durante su consulta? }\end{array}$ & $555(90,9)$ & $572(93,7)$ \\
\hline $\begin{array}{l}\text { ¿Transcurre mucho tiempo entre el momento de atenderlo y la hora } \\
\text { acordada? }\end{array}$ & $472(77,3)$ & $537(88,0)$ \\
\hline $\begin{array}{l}\text { ¿El tiempo de espera entre cita y cita se acopla a su disponibilidad y necesidad } \\
\text { de tratamiento? }\end{array}$ & $551(90,3)$ & $565(92,6)$ \\
\hline ¿El estudiante lo ha dejado durante un tiempo prolongado solo en el cubículo? & $199(32,6)$ & $127(20,8)$ \\
\hline $\begin{array}{l}\text { ¿El trato recibido durante una llamada telefónica para citarlo ha sido } \\
\text { amigable? }\end{array}$ & $545(70,9)$ & $588(96,3)$ \\
\hline $\begin{array}{l}\text { ¿El estudiante lo ha llamado después de realizarle un tratamiento para saber } \\
\text { cómo es su estado de salud? }\end{array}$ & $433(70,9)$ & $524(85,9)$ \\
\hline Factores relacionados con transmisión del conocimiento estudiante-paciente & $\begin{array}{c}\text { T0 } \\
\text { Frecuencia } \\
(\%) \\
\end{array}$ & $\begin{array}{c}\mathrm{T1} \\
\text { Frecuencia (\%) }\end{array}$ \\
\hline $\begin{array}{l}\text { ¿El estudiante lo atiende solo como un requisito de su clínica o como un ser } \\
\text { integral? }\end{array}$ & $151(24,7)$ & $123(20,1)$ \\
\hline $\begin{array}{l}\text { ¿Notó usted al estudiante de odontología seguro durante el procedimiento } \\
\text { que le estuvo realizando mientras estaba solo? }\end{array}$ & $557(91,3)$ & $553(90,6)$ \\
\hline $\begin{array}{l}\text { ¿Observó al estudiante inseguro al hablar con usted y atenderlo y al estar con } \\
\text { el docente? }\end{array}$ & $176(28,8)$ & $152(24,9)$ \\
\hline $\begin{array}{l}\text { ¿El estudiante le explica sobre procedimiento o tratamiento antes de } \\
\text { realizarlo? }\end{array}$ & $556(91,1)$ & $564(92,4)$ \\
\hline
\end{tabular}




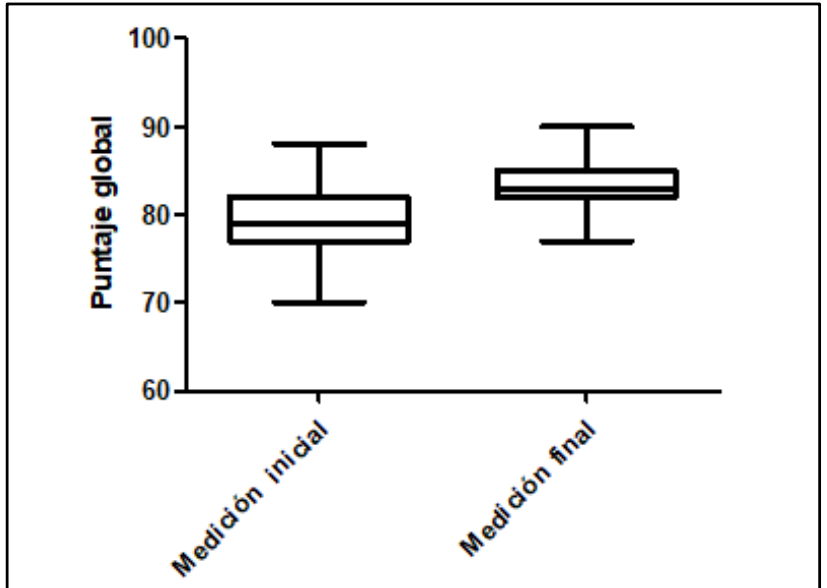

Figura 1. Percepción inicial (T0) y final del paciente (T1) con respecto al servicio de odontología ofertado por el estudiante de odontología.

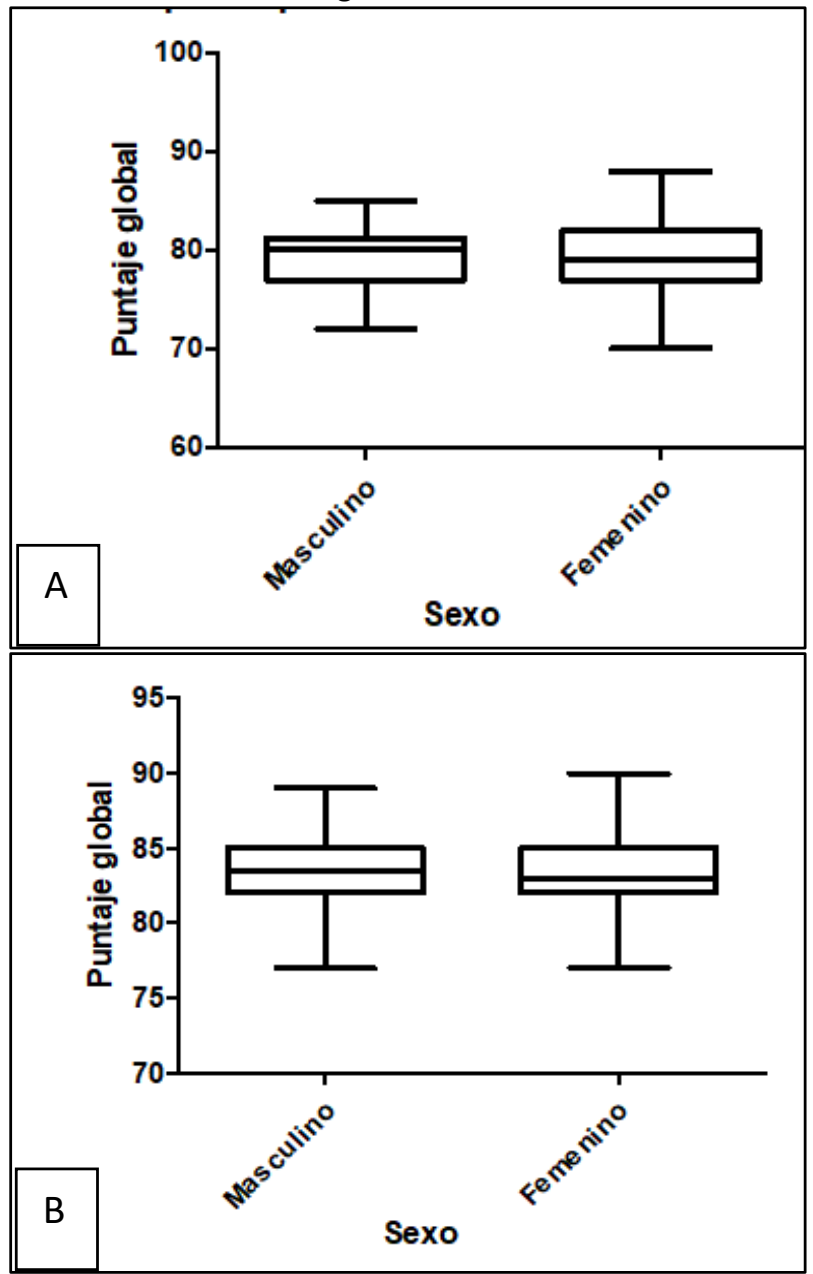

Figura 2. Percepción del paciente con respecto al servicio de odontología ofertado por el estudiante de odontología según el sexo. A. Percepción inicial (T0). B. Percepción final (T1).
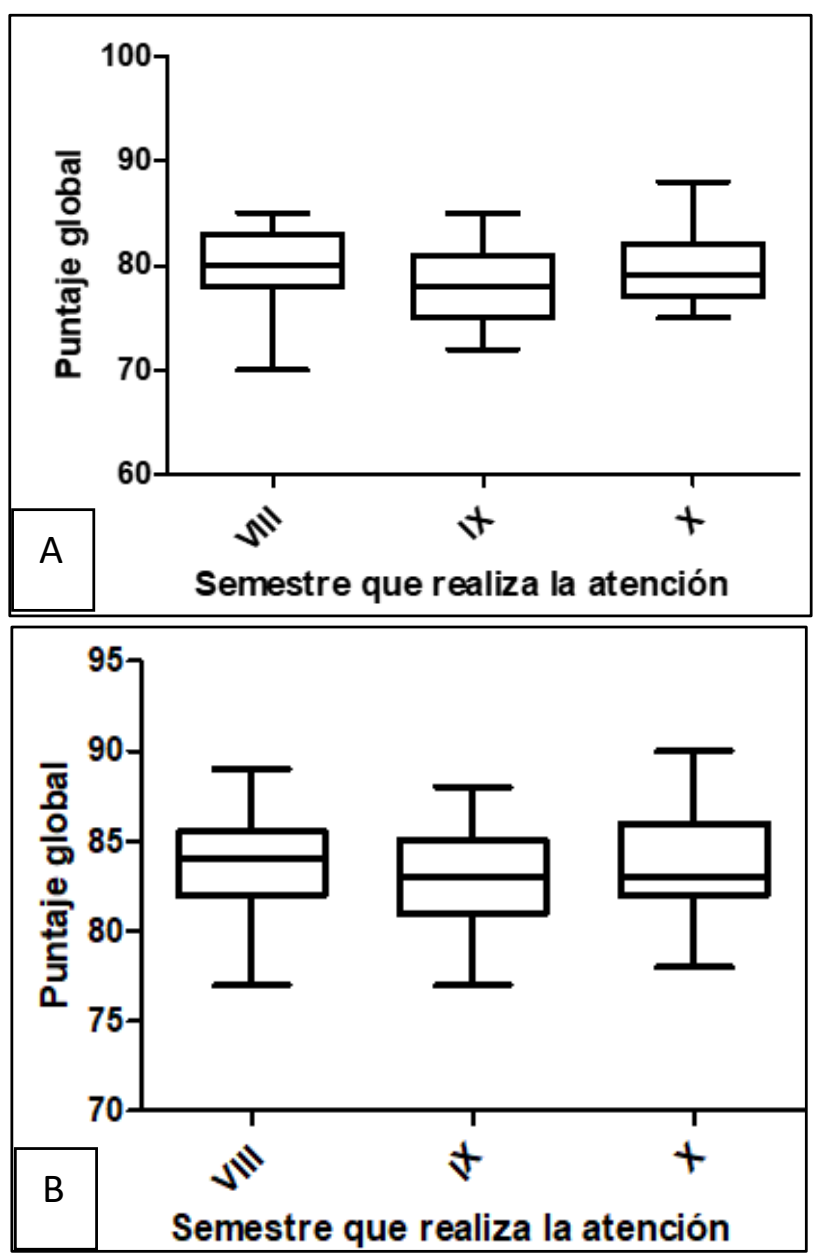

Figura 3. Percepción del paciente con respecto al servicio de odontología ofertado por el estudiante de odontología según el semestre. A. Percepción inicial (TO). B. Percepción final (T1).

\section{DISCUSIÓN}

A la luz de la revisión bibliográfica, este corresponde a uno de los pocos estudios que evalúan la percepción del paciente con respecto a la atención ofrecida por estudiantes de odontología en la práctica clínica. La gran mayoría de investigaciones en la materia examinan esta atención cuando es prestada por parte de odontólogos que han finalizado sus estudios y se encuentran graduados. Por tanto, las comparaciones entre los estudios resultan limitadas en este sentido.

En este trabajo se reporta que la percepción de los pacientes sobre la atención recibida al finalizar su tratamiento odontológico es mejor cuando son atendidos por estudiantes de semestres más 
avanzados. Es probable que esto obedezca a una mayor seguridad con respecto a estudiantes que se encuentran a punto de culminar su carrera profesional dados un mayor dominio del conocimiento y un mejor manejo del contexto clínico. Al respecto, Elizondo-Elizondo et $a l^{11} \mathrm{y}$ Viscaíno ${ }^{12}$ reportan que existen aspectos relacionados con la percepción y satisfacción del paciente durante una atención clínica como empatía, cortesía, amabilidad, tranquilidad y comunicación, los cuales van mejorando o se van adquiriendo durante el desarrollo del tratamiento del paciente, de forma que se logra modificar esta percepción de parte de los pacientes ${ }^{13,14}$.

Este transcurrir del tiempo ofrece oportunidades al paciente para cambiar su percepción. Así, tanto si la situación es positiva como si es negativa para el estudiante en las primeras consultas, se impone el deber de la autoevaluación y coevaluación de la atención prestada en aras de ofertar un servicio con calidad. En ese orden de ideas, Lang ${ }^{15}$ reporta que la percepción que poseen los usuarios del servicio y atención odontológica brindada en las instalaciones de la Universidad de San Carlos de Guatemala responde precisamente a las actitudes personales que presenta el estudiante con el paciente al inicio, durante y al final del tratamiento realizado ${ }^{16}$.

Se pudo establecer que la percepción del paciente es modificada por diversos factores como la personalidad, el ambiente, la supervisión por parte del docente y la presentación personal del estudiante, un hallazgo que coincide con lo reportado por Orenuga et $a l^{17}$, quienes evaluaron la percepción de insatisfacción de usuarios asistentes al programa de Odontologia de la Universidad de Lagos en Nigeria. En este caso, dicha percepción estuvo íntimamente relacionada con factores y condiciones físicas propias del lugar donde se brindaba la atención, entre los cuales se destacan el fluido eléctrico, la constancia en el suministro de agua y la realización de tratamientos por estudiantes a pacientes sin la supervisión por parte del docente asignado.

Asimismo, considerarse el paciente como un requisito de la clínica y otros comportamientos que se dan de forma natural entre los estudiantes como el préstamo de instrumental odontológico durante la atención odontológica, contestar el celular o realizar otra actividad con guantes son aspectos que modificaron la percepción del paciente en el tiempo, aunque con bajos valores ${ }^{1}$. Estos comportamientos podrían explicarse por la confianza que el estudiante va adquiriendo con su paciente, así como por el hecho de que el docente no puede permanecer de manera constante junto al estudiante, lo que conllevaría a subestimar la percepción del paciente frente a la atención odontológica recibida y confundir el objetivo de la consulta en relación con la satisfacción de las necesidades del paciente en primer lugar. En este sentido cabe anotar que el estudiante debe guardar precaución en no descuidar aspectos fundamentales en la atención odontológica de los pacientes como el respeto.

Lora et $a l^{10}$, en un estudio en una universidad de naturaleza privada en la ciudad de Cartagena, Colombia, reportan que la mayoría de los usuarios $(98,9 \%)$ manifestaron satisfacción y conformidad respecto a ciertos indicadores como bioseguridad (la higiene del instrumental y la organización del lugar de trabajo). Esto coincide con el presente estudio, en donde el uso de barreras de bioseguridad y el orden del instrumental y del área de trabajo fueron variables que presentaron una excelente percepción, de $94,1 \%$ y $92,7 \%$ respectivamente. De tal forma, considerando que el estudio se llevó a cabo en una clínica universitaria de carácter público, se rompe el mito de la insatisfacción y la mala calidad ligado a la naturaleza de las instituciones de salud.

Dentro de otros factores relacionados con la percepción de la atención clínica, dejar al paciente solo en la unidad odontológica durante un tiempo prolongado hace que la percepción sea menos favorable en esta variable $(20,8 \%)$. Esto coincide con lo reportado por Adeniyi et $a l^{18}$ en su estudio sobre satisfacción de los pacientes que asisten a la Clínica Dental de la Universidad Estatal de Lagos, Nigeria, en donde el largo tiempo de espera fue el elemento que menos les gustó a los encuestados sobre los servicios. Asimismo, se debe hacer énfasis a los estudiantes sobre la importancia de la adecuada y completa disposición de todos los materiales y elementos necesarios para la atención a los 
pacientes; de lo contrario, estos últimos pueden notar falta de organización y planificación. Dicha posibilidad se agrava si no se adquieren estos instrumentos en las clínicas, comprometiendo la imagen de la institución y generando una inadecuada voz a voz entre los pacientes que no fomentaría el aumento en la demanda, situación que finalmente perjudicaría a los mismos estudiantes. Por ende, debe advertirse al paciente sobre algunas situaciones que se experimentan en semestres clínicos inferiores y que marcan una diferencia en la atención ofrecida, lo que no implica dejar de gozar de calidez humana y de una atención integral y respetuosa en cualquier instancia de aprendizaje. En definitiva, esta variable no condiciona la percepción de los pacientes.

Por otra parte, López-Portilla et $a l^{5}$ en Medellín, Colombia, identificaron ciertos factores que influyen en la satisfacción de los pacientes y que no estaban directamente relacionados con el semestre académico del estudiante, sino con situaciones externas (problemas de orden público dentro de la institución) que terminan perjudicando la calidad, la continuidad y la culminación del tratamiento principalmente. A su vez, se amerita una evaluación permanente de la atención prestada a los pacientes por las diferentes facultades de Odontología del país durante todos los momentos de contacto del servicio odontológico, controlando los riesgos de una prestación mecánica y deshumanizada, que únicamente se concentre en la salud bucal del paciente y descuide otros aspectos fundamentales en la relación psicosocial odontólogo-paciente, queja principal reportada en el Sistema General de Seguridad Social en Salud ${ }^{19}$. En ese orden de ideas, estos resultados podrían conllevar a replantear los modelos de atención prestados desde la academia de manera que se incluya la evaluación de estas percepciones en función de la retroalimentación del proceso de atención clínica.

Las pacientes de sexo femenino presentan en este estudio una mejor percepción frente a la atención ofrecida por los estudiantes. Autores como Derose et $a l^{20}$, Betin Portacio et $a l^{21}$ y Giraldo Molina et $a l^{22}$ confirman en sus estudios que el sexo al que pertenece el paciente se encuentra estrechamente relacionado con la percepción de satisfacción debido a que las mujeres se asocian en mayor proporción a experiencias positivas durante el tratamiento recibido con respecto a los usuarios hombres. Frente a esta variable, se ha señalado cómo las mujeres, en comparación con los hombres, comprenden mejor las situaciones en el manejo de una enfermedad, la atención del servicio recibido y las fallas en los tratamientos, así como algunos detalles relacionados con la infraestructura de los consultorios ${ }^{23}$.

De igual modo, el sexo del profesional de la salud influye directamente en la relación que se instaura con el paciente en el momento de brindar la atención ${ }^{24}$. Delgado et $a l^{25}$, por ejemplo, indican que los pacientes prefieren ser atendidos por médicas debido a que ellas ofrecen consultas más duraderas, facilitan más información, muestran una forma de comunicación más sutil y gentil, y brindan la sensación de que se puede participar de manera más activa durante la consulta. Además, se encontró que la mayor parte de los pacientes que acuden a las clínicas en donde la atención es brindada por estudiantes pertenecen al sexo femenino $(59,4 \%)$, lo que concuerda con lo reportado por Hincapié et a ${ }^{26}$, Agudelo et $a{ }^{27}$, Kfouri et $a{ }^{28}$ y Garbin et a ${ }^{29}$, quienes asocian el sexo femenino del estudiante de odontología a las expresiones de atención, cuidado, delicadez y paciencia, mientras que en los hombres se perciben expresiones de seguridad, cuidado y fuerza, favoreciendo de manera positiva o negativa la relación entre el personal que brinda la atención y el paciente ${ }^{30,31}$.

Dado lo anterior, cabe resaltar que en este estudio más de la mitad de los estudiantes correspondían al sexo femenino, lo que influye en el comportamiento de los resultados aquí reportados y, en últimas, correspondería a una limitación de la investigación. Por lo tanto, valdría la pena adelantar más estudios con poblaciones estratificadas por sexo para realizar apreciaciones más contundentes al respecto. Otra limitación tiene que ver con la evaluación de estas percepciones en solo dos momentos de la atención, lo que no permitiría realizar análisis más precisos y conclusivos. Sin embargo, los resultados son alentadores y motivantes para las diferentes escuelas de odontología al comprobar que las competencias de estos futuros profesionales se están adquiriendo. 
Por último, esta investigación, mediante sus hallazgos, da una idea de cómo las facultades de Odontología en Colombia y en el mundo deben concentrar su atención en la adquisición de competencias, tanto disciplinares como humanísticas y sociales, que ayuden a enfrentar los problemas y las necesidades de una sociedad que requiere cada vez más una atención verdaderamente integral y holística, que mire "más allá de la boca"32. De aquí la importancia del fortalecimiento de asignaturas o temáticas al respecto en los planes de estudio de profesionales de la salud, buscando generar un impacto positivo en el medio y cumpliendo con la misión de educación, extensión y proyección social del servicio hacia la comunidad en aras de contribuir a mejorar su calidad de vida.

\section{CONCLUSIÓN}

La percepción de los pacientes frente a la atención odontológica ofrecida por estudiantes se encuentra influenciada no solo por factores relacionados con la infraestructura de una institución, sino también por factores propios del estudiante como el sexo al que pertenece $y$ el semestre. Esto se refleja directamente sobre la interacción estudiantepaciente, que conlleva a satisfacción de la atención recibida y éxitos en los tratamientos odontológicos realizados.

Estos hallazgos podrían dar pie a estudiar y seguir los posibles factores que comprometan la imagen percibida por la comunidad sobre la atención odontológica ofertada en las clínicas adscritas a instituciones universitarias, con miras a generar una mayor afluencia de pacientes mejorando indicadores de morbilidad bucal a bajos costos y abordando las limitaciones en la consecución de estos por parte del estudiantado, inconformidad muchas veces presentada en los diferentes programas de odontología de Colombia y el mundo.

\section{DECLARACIÓN SOBRE CONFLICTOS DE INTERÉS}

Los autores del presente estudio declaran no tener conflictos de intereses con ninguna parte involucrada.

\section{CONTRIBUCIÓN DE LOS AUTORES}

Primer autor: diseño metodológico, análisis estadístico, trabajo de campo, redacción del borrador del artículo y elaboración final del manuscrito.

Segundo autor: redacción del borrador del artículo y elaboración final del manuscrito.

Tercer autor: redacción del borrador del artículo y elaboración final del manuscrito.

Cuarto autor: diseño metodológico, análisis estadístico, trabajo de campo.

Quinto autor: diseño metodológico, análisis estadístico, trabajo de campo.

\section{REFERENCIAS BIBLIOGRÁFICAS}

1. Yépez-Chamorro MC, Ricaurte-Cepeda M, Jurado DM. Calidad percibida de la atención en salud en una red pública del municipio de Pasto, Colombia. Univ Salud. 2018; 20(2): 97-110. Doi: http://dx.doi.org/10.22267/rus.182002.114.

2. Torres-Gonzáles GC, León-Manco RA. Nivel de satisfacción de los pacientes atendidos en el Servicio de Ortodoncia de una Clínica Dental Docente peruana. Rev Estomatol Herediana. 2015; 25(2): 122-32.

Doi: https://doi.org/10.20453/reh.v25i2.2458.

3. Donabedian A. Evaluating the Quality of Medical Care.The Milbank Q. 2005; 83(4): 691-729.

Doi:

https://doi.org/10.1111/j.1468-

0009.2005.00397.x.

4. Varo J. Gestión estratégica de la calidad en los servicios sanitarios: un modelo de gestión hospitalaria. Madrid: Ediciones Díaz de Santos; 1994.

5. López-Portilla JE, Pilataxi-Sánchez S, RodríguezEscobar LD, Velásquez-Rivera AC, López-Blandón MA, Martínez-Delgado CM et al. Determinantes de la satisfacción de la atención odontológica en un grupo de pacientes atendidos en la Clínica del Adulto de la Facultad de Odontología de la Universidad de Antioquia. Revista Gerencia y Políticas de Salud 2013; 12(24): 209-25. Disponible en: 
http://www.scielo.org.co/pdf/rgps/v12n24/v12n24 a13.pdf.

6. Torres-Quintana MA, Romo OF. Bioética y ejercicio profesional de la odontología. Acta Bioeth. 2006; 12(1): 65-74. Doi: http://dx.doi.org/10.4067/S1726-

569X2006000100010.

7. Silva-Fhon J., Ramón-Cordova S., VergarayVillanueva S., Palacios-Fhon V., Partezani-Rodrigues R. Percepción del paciente hospitalizado respecto a la atención de enfermería en un hospital público. Enferm Univ. 2015; 12(2): 80-7. Doi: http://dx.doi.org/10.1016/j.reu.2015.04.001.

8. Oketade IO, Osiro O, Ibiyemi ST, Ibiyemi O. Perception of patients attending a tertiary hospital in Nigeria about good dental practice: a pilot study. Níger J Clin Pract. 2013; 16(04): 478-82. Doi: https://doi.org/10.4103/1119-3077.116893.

9. Henríquez-Tejo RB, Cartes-Velásquez RA. Percepciones de la población respecto al cirujano dentista. Odontoestomatología. 2016; 18(27): 1624. Disponible en: http://www.scielo.edu.uy/scielo.php?pid=S168893392016000100003\&script=sci_arttext.

10. Lora-Salgado IM, Tirado-Amador LR, MontoyaMendoza JL, Simancas-Pallares MÁ. Percepción de satisfacción y calidad de servicios odontológicos en una clínica universitaria de Cartagena, Colombia. Rev Nac Odontol. 2016; 12(23): 31-40. Doi: https://doi.org/10.16925/od.v12i23.1378.

11 Elizondo-Elizondo J, Quiroga-García MA, Palomares-Gorham PI, Martínez-González GI. La satisfacción del paciente con la atención técnica del servicio odontológico. Revista Salud Pública y Nutrición 2011; 12(1). Disponible en: https://www.medigraphic.com/cgi-

bin/new/resumen.cgi?IDARTICULO=29820.

12. Viscaíno Salem KM. Grado de satisfacción de los usuarios del servicio de la Clínica Integral Odontológica de la Universidad de las Américas. [Trabajo de grado para título de odontóloga]. Quito: Universidad de las Américas. Facultad de Odontología; 2016.
13. Lara-Flores N, López-Cámara V, Morales-Estrella SL, Cortés-Velázquez E. Satisfacción de usuarios de dos servicios odontológicos en Tláhuac, Distrito Federal. Rev ADM. 2000; 57(2): 45-49. Disponible en: $\quad$ https://www.medigraphic.com/cgi$\mathrm{bin} / \mathrm{new} /$ resumen.cgi?IDARTICULO=2623

14. Estabrook B, Zapka J, Lubin H. Consumer Perceptions of Dental Care in the Health Services Program of an Educational Institution. J Am Dent Assoc 1980; 100(4): 540-3. Doi: https://doi.org/10.14219/jada.archive.1980.0150.

15. Lang J. Percepción del paciente que asiste a las clínicas de la facultad de odontología de la universidad de san Carlos de Guatemala, acerca del servicio y atención odontológica, otorgados por los estudiantes, personal docente y administrativo. [Trabajo de grado para título de cirujana dentista]. Guatemala: Universidad de San Carlos. Facultad de Odontología; 2004.

16. Gopalakrishna P, Munnaleneni V. Influencing satisfaction for dental services. J Health Care Mark. 1993; 13(1): 16-22. Disponible en: https://www.ncbi.nlm.nih.gov/pubmed/10126026.

17. Orenuga OO, Sofola OO, Uti OO. Patient satisfaction: a survey of dental outpatients at the Lagos University Teaching Hospital, Nigeria. Nig Q J Hosp Med. 2009; 19(1): 47-52. Doi: https://doi.org/10.4314/nqjhm.v19i1.50208.

18. Adeniyi AA, Adegbite KO, Braimoh MO, Ogunbanjo BO. Factors affecting patient satisfaction at the Lagos State University Teaching Hospital Dental Clinic. Afr J Med Sci. 2013 Mar; 42(1): 25-31. Disponible en: https://www.ncbi.nlm.nih.gov/pubmed/23909091. 19. López-Soto OP, Cerezo-Correa M del P, PazDelgado AL. Variables relacionadas con la satisfacción del paciente de los servicios odontológicos. RGYPS. 2010; 9(18). Disponible en: https://revistas.javeriana.edu.co/index.php/gerepo Isal/article/view/2639/1901

20. Derose KP, Hays RD, McCaffrey DF, Baker DW. Does physician gender affect satisfaction of men and women visiting the emergency department? J Gen 
Intern Med. 2001 Apr; 16(4): 218-26. Doi: https://doi.org/10.1046/j.1525-

1497.2001.016004218.x.

21. Betin Portacio A, Guardela Simancas A, Infante Franco K, Díaz Caballero A. Satisfacción de los pacientes respecto a calidad de la atención odontológica en una institución pública universitaria de la ciudad de Cartagena. Duazary. 2009; 6(2): 95$101 . \quad$ Disponible en: http://revistas.unimagdalena.edu.co/index.php/du azary/article/view/674.

22. Giraldo Molina AM, Berbesi Fernández DY. Key factors in the perception of the quality of dental services provided by undergraduate students. Rev Fac Odontol Univ Antioq. 2017; 28(2): 311-26. Doi: http://dx.doi.org/10.17533/udea.rfo.v28n2a5.

23. Smith MK, Dundes L. The implications of gender stereotypes for the dentist-patient relationship. J Dent Educ 2008; 72(5): 562-70. Disponible en: https://www.ncbi.nlm.nih.gov/pubmed/18451079.

24. Roter DL, Hall JA, Aoki Y. Physician Gender Effects in Medical Communication: A Meta-Analytic Review. JAMA. 2002; 288(6): 756-64. Doi: https://doi: 10.1001/jama.288.6.756.

25. Delgado A, López-Fernández LA. Práctica profesional y género en atención primaria. Gac Sanit. 2004; 18(4): 112-7. Disponible en: http://scielo.isciii.es/scielo.php?script=sci_arttext\& pid=S0213-91112004000400018\&Ing=es.

26. Hincapié A, Carvajal Á, Sánchez LA, Arias Ó. Satisfacción de los usuarios frente a la calidad de la atención en el programa de odontología integral del adolescente y ortodoncia de la facultad de odontología de la universidad de Antioquia. Rev Fac Odontol Univ Antioq. 2004; 15(2): 5-11. Disponible en:

https://revistas.udea.edu.co/index.php/odont/artic le/view/3238.

27. Agudelo AA, Valencia LY, Oullón E, Betancur LG, Restrepo LM, Peláez LV. Satisfacción y factores asociados en estudiantes usuarios del servicio odontológico de la institución prestadora de servicios de salud "IPS Universitaria" (Medellín). Rev Fac Odontol Univ Antioq. 2008; 19(2): 13-23. Disponible

en http://www.scielo.org.co/scielo.php?script=sci_artt ext\&pid=S0121246X2008000100003\&lng=en.

28. Kfouri MG, Moyses SJ, Moyses ST. Women's motivation to become dentists in Brazil. J Dent Educ 2013; 77(6): 810-816. Disponible en: https://www.ncbi.nlm.nih.gov/pubmed/23740918.

29. Garbin AJI, Garbin CAS, Tiano AVP, Carvalho ML, Fagundes ACG. Marketing em Odontologia: a percepção do cliente em relação ao serviço odontológico de clínica privada Rev Odontol UNESP 2008; 37(2): 197-202. Disponible en: https://www.revodontolunesp.com.br/journal/rou /article/588018867f8c9d0a098b4cae\#nav.

30. Kfouri MG, Simone Tetu S, Gabardo MC, Nascimento AC, Saulo Vinicius R, Moysés SJ. The feminization of dentistry and the perceptions of public service users about gender issues in oral health. Ciênc. Saúde coletiva. 2019; 24(11). Doi: https://doi.org/10.1590/1413-

812320182411.00832018 .

31. McKay JC, Quiñonez CR. The feminization of dentistry: implications for the profession. J Can Dent Assoc. 2012; 78: c1. Disponible en: https://www.ncbi.nlm.nih.gov/pubmed/22322017.

32. Díaz Cárdenas S, Tirado Amador LR, Madera Anaya MV. Odontología con enfoque en salud familiar. Rev Cubana Salud Pública. 2014; 40(3): 397$405 . \quad$ Disponible en: https://www.medigraphic.com/pdfs/revcubsalpub/ csp-2014/csp143j.pdf 